Research letter

\title{
Blood pressure load in adolescent boys with different circadian systolic and diastolic blood pressure patterns
}

\author{
Anastasia Sheveleva, Sergey Klauchek, Mikhail Ledyaev \\ Volgograd State Medical University, Volgograd, Russia
}

Received 11 December 2017, Revised 18 May 2018, Accepted 19 September 2018

C 2017, Sheveleva A., Klauchek S., Ledyaev M.

(C) 2017, Russian Open Medical Journal

\begin{abstract}
The present study was designed to analyze the occurrence of increased blood pressure load in adolescent boys with different circadian blood pressure patterns. Healthy adolescent boys with normal values of both office and mean ambulatory systolic blood pressure (SBP) and diastolic blood pressure (DBP) had 3 types of circadian blood pressure profile: "dippers", "non-dippers", "over-dippers". The occurrence of increased blood pressure load was the highest in the groups with a nocturnal over-dipping pattern for both SBP and DBP.
\end{abstract}

Keywords: blood pressure load, non-dippers, over-dippers, dippers, adolescents.

Cite as Sheveleva A, Klauchek S, Ledyaev M. Blood pressure load in adolescent boys with different circadian systolic and diastolic blood pressure patterns. Russian Open Medical Journal 2018; 7: e0413.

Correspondence to Anastasia Sheveleva.E-mail: a.m.ledyaeva@gmail.com.

\section{Introduction}

Ambulatory blood pressure monitoring (ABPM) plays an important role in the management of patients with arterial hypertension $(\mathrm{AH})$ [1-3]. This method of diagnosis provides information about average daytime, nighttime and 24-hours values of systolic, diastolic and pulse blood pressure, blood pressure load (BP load) and nocturnal decline of blood pressure, so it allows studying in detail the circadian BP pattern [1-4].

BP load is the percentage of readings above the threshold during 24 hours [1]. In the pediatric population, the threshold is the value of $95^{\text {th }}$ percentile according to sex, age and height [5]. BP load should be less than $25 \%$. Patients with BP load more than $50 \%$ have an increased risk of target organ damage [6]. The values of BP load that exceeded $25 \%$, but are less than $50 \%$, in combination with increased office BP act as one of the criteria of prehypertension [5], but in case of normal values of both office and mean ambulatory systolic blood pressure (SBP) or diastolic blood pressure (DBP), it is regarded as a risk factor of $\mathrm{AH}$ development.

Bood pressure (BP) has a circadian rhythm characterized by a decline during sleep at night by 10 to $20 \%$ relative to daytime values [7]. There are four types of diurnal BP patterns based on the value of diurnal BP fall: individuals with 10-20\% decline are called "dippers"; those who have $>20 \%$ decline are called "extreme dippers" or "over-dippers"; patients with $<10 \%$ decline are "nondippers" and with $<0 \%$ decline are "risers" or "night-peakers" [8].

Several studies reported that abnormal dipping status in adults is associated with obesity [9], development of chronic kidney disease [10] and increased risk of cardiovascular diseases [11], but there is lack of data about this problem in the pediatric population. Furthermore, it is well-known that essential $\mathrm{AH}$ is a current epidemic problem in adults and BP abnormalities have their origins in childhood, so it confirms the importance of early diagnosis and prevention strategies [3].

The goal of this study was to describe the occurrence of increased BP load in adolescent boys with different circadian BP patterns.

\section{Material and Methods}

Figure 1 shows the scheme of study design. We recruited 190 boys 12-17 years old. The inclusion criteria were office BP levels (in three separate measurements) and 24-h SBP and DBP levels less than 95th percentile according to sex, height and age. Adolescents with congenital heart and vessel disease, arrhythmia, acute and chronic kidney disease and body mass index (BMI) less than the 10th percentile and more than the $90^{\text {th }}$ percentile according to sex and age were excluded from the study.

We performed ABPM using oscillometric device BPLab (level of accuracy A/A [12]) with the appropriate size cuff that was applied to the child's nondominant arm. The device was programmed to record BP every 15 min during daytime and every 30 min during sleep. The participants maintained a diary and indicated sleep and wake times, physical activities, stressful situations, dizziness, bad quality of sleep or sleepiness. We accepted only ABPM profiles with at least $70 \%$ of recordings being obtained at least every $30 \mathrm{~min}$.

We analyzed the nocturnal SBP and DBP fall, systolic and diastolic BP load based on data of ABPM.

According to $A B P M$, we divided the participants into 3 groups depending on the value of diurnal BP fall: "dippers" $(10-20 \%$ nocturnal decline of BP); "over-dippers" ( $>20 \%$ nocturnal decline of BP); "non-dippers" ( $<10 \%$ nocturnal decline of $B P$ ). 
Table 1. Basic characteristics of study participants

\begin{tabular}{lcccccccc}
\hline Parameter & $L Q$ & Me & UQ & $I Q R$ & Min & Max & Mean & $S D$ \\
\hline Age, years & 13 & 15 & 16 & 3 & 12 & 17 & 14.6 & 1.6 \\
Height, m & 1.62 & 1.70 & 1.77 & 0.15 & 1.42 & 1.92 & 1.70 & 0.1 \\
Weight, kg & 49 & 56 & 64 & 15 & 31 & 90 & 57 & 11 \\
$\mathrm{BMI}, \mathrm{kg} / \mathrm{m}^{2}$ & 18 & 19.1 & 21 & 3 & 14.7 & 25.1 & 20 & 2.4 \\
$\mathrm{SBP}_{\text {day }}, \mathrm{mmHg}$ & 117 & 122 & 125 & 8 & 103 & 131 & 121 & 6.3 \\
$\mathrm{SBP}_{\text {night }}, \mathrm{mmHg}$ & 101 & 106 & 111 & 10 & 65 & 123 & 105 & 8.6 \\
$\mathrm{DBP}_{\text {day }}, \mathrm{mmHg}$ & 67 & 70 & 73 & 6 & 53 & 86 & 70 & 5 \\
$\mathrm{DBP}_{\text {night }}, \mathrm{mmHg}$ & 53 & 57 & 61 & 8 & 44 & 70 & 57 & 5 \\
$\mathrm{PBP}_{\text {day }}, \mathrm{mmHg}$ & 46 & 51 & 55 & 9 & 34 & 69 & 51 & 6.5 \\
$\mathrm{PBP}_{\text {night }}, \mathrm{mmHg}$ & 45 & 51 & 53 & 8 & 28 & 73 & 49 & 6.7 \\
\hline
\end{tabular}

$L Q$, lower quartile; Me, median; UQ, upper quartile; IQR, interquartile range; Min, minimum; Max, maximum; SD, standard deviation; BMI, body mass index; SBP, systolic blood pressure; DBP, diastolic blood pressure; PBP, pulse blood pressure.

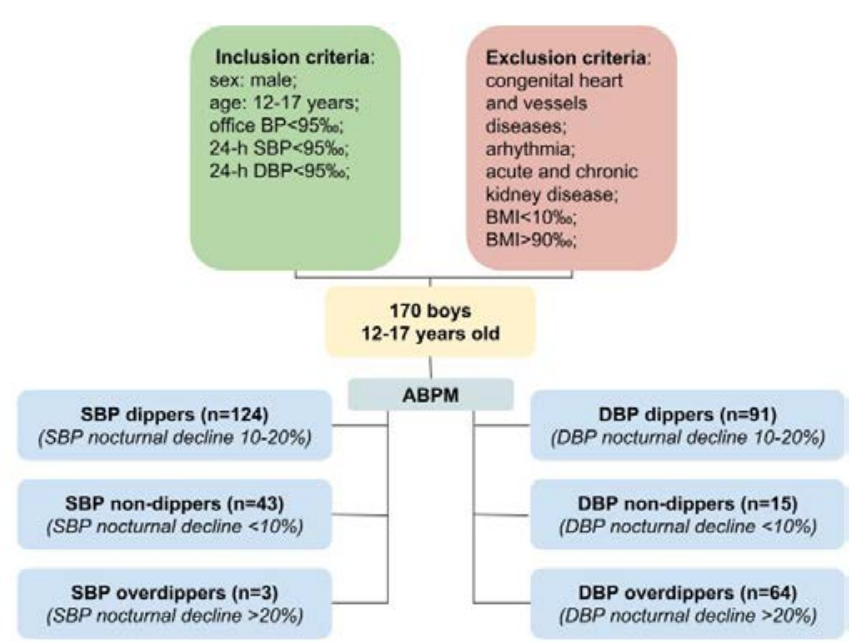

Figure 1. Scheme of study design.

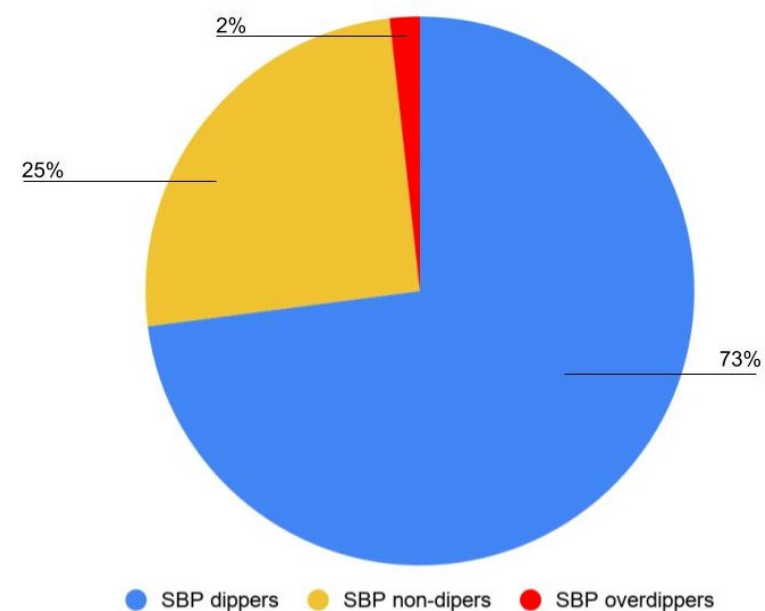

Figure 2. Prevalence of diurnal blood pressure patterns of SBP in adolescent boys $12-17$ years.

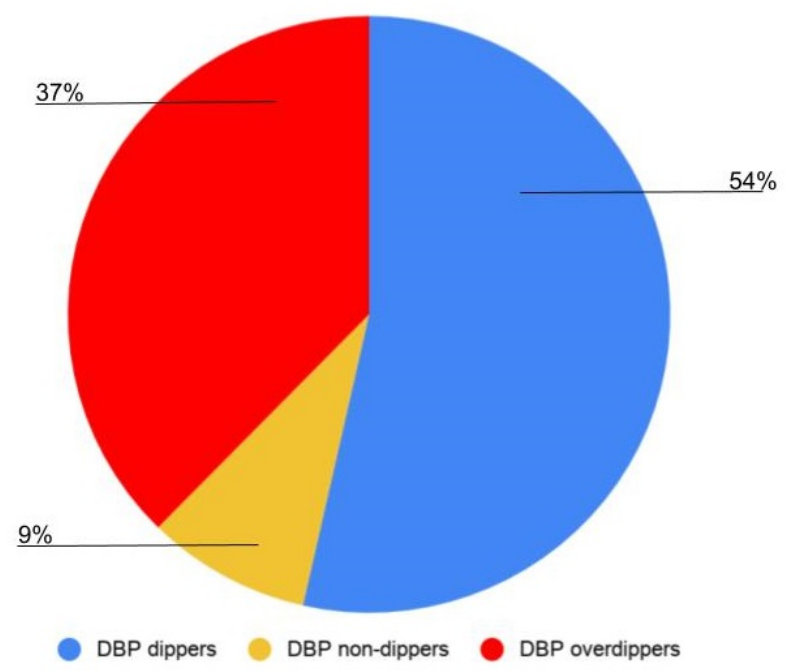

Figure 3. Prevalence of diurnal blood pressure patterns of DBP in adolescent boys $12-17$ years.

The study was approved by the regional scientific ethical committee. All the participants and their parents received detailed information about the procedure of measurements, were instructed how to stop readings if there is severe discomfort and signed a written consent.

Statistical data processing was done with Microsoft Excel 2007 (Microsoft, USA) and Statistica 6.0 (StatSoft, USA). We used Shapiro-Wilk's $\mathrm{W}$ test to determine the type of distribution. All data are shown as median (Me), mean, maximum (max), minimum (min), interquartile range (IQR) and standard deviation (SD). The frequency of occurrence is shown in percents. We compared qualitative variables using the Pearson chi-square test or the Fisher exact test when the calculated sample size was $<5$.

\section{Results}

Weight, daytime and nighttime DBP, daytime and nighttime PP followed normal distribution due to the Shapiro-Wilk's $W$ test. BMI, height, daytime and nighttime SBP followed a skewed distribution. Table 1 reports the values of anthropometric measures and ABPM results in studied group of boys.

Figure 2 shows the percentage occurrence of three circadian patterns of SBP and DBP. Due to the level of SBP dipping we found that $73 \%(n=124)$ of boys had normal circadian BP profile (SBP dippers), loss of the nocturnal decline of SBP (SBP non-dippers) was found in $25 \%(n=43)$ of participants and $2 \%(n=3)$ had an excessive fall in SBP at night (SBP over-dippers). The analysis of the DBP dipping showed different results: $54 \% \quad(n=91)$ of the participants had from 10 to $20 \%$ decline in night to day diastolic BP (DBP dippers), 37\% ( $n=64)$ had an excessive fall in DBP at night (DBP over-dippers), 9\% ( $n=15)$ had loss of the nocturnal decline of DBP (DBP non-dippers) (Figure 3). The occurrence of different BP patterns was significantly different for SBP and DBP (Chisquare $=75.5, \mathrm{df}=2, \mathrm{p}<0.001$ ).

Then we calculated the percentage occurrence of increased $\mathrm{BP}$ load in boys with different diurnal SBP and DBP patterns (Figures 4 and 5). In the groups "SBP dippers" and "SBP non-dippers" only $19 \%$ (24/124 and 8/43, respectively) of boys had BP load more 
than $25 \%$, but less than $50 \%$. The highest percent of boys $-66 \%$ $(2 / 3)$ with increased BP load was in the group "SBP over-dippers". The percentage occurrence of increased BP load was 16\% (15/91) in the "DBP dippers", 13\% (2/15) in the "DBP non-dippers" and $27 \%(17 / 64)$ in "DBP over-dippers".

The Pearson chi-square test and the Fisher exact test showed the significant difference in the percentage occurrence of increased BP load only between "SBP over-dippers» and "SBP dippers» (Fisher exact $p=0.03$, Chi-square $=7.2, d f=1, p=0.007$ ).

\section{Discussion}

In this study of adolescent boys with normal values of both office and mean ambulatory SBP and DBP we have found 3 types of circadian blood pressure profile: "dippers", "non-dippers" and "over-dippers". The type "night-peakers" was absent, so it means that in the study group there were no participants with nighttime hypertension. The most frequent abnormal type of SBP rhythm was a non-dipping pattern, on the contrary, for DBP it was the over-dipping pattern with excessive fall at night. The occurrence of increased BP load was the highest in the groups with the nocturnal over-dipping pattern for both SBP. The main limitation of the present study is the small size of "SBP over-dippers" group, so it is necessary to continue the study and to repeat the analysis in a bigger sample of participants. In available literature, there are few data on the prevalence of different dipping patterns in the general pediatric population. There are some studies that reported significant association between nocturnal non-dipping pattern and obesity [13], left ventricular hypertrophy [14] and elevation of carotid intima-media thickness [15]. On the contrary, there is lack of data about the significant effects of extreme dipping status on health outcomes in the pediatric population. A lot of exogenous environmental 24-hours cycles and endogenous circadian rhythms contribute to the typical rhythm of BP. Circadian rhythm of BP is connected with vagal tone, sympathetic nervous system activity and plasma adrenalin/noradrenaline and atrial natriuretic peptide concentrations. Even the endothelial function has its own circadian profile that we can see in dynamic concentrations of nitric oxide and endothelin-1 during a day [7]. So it seems to be obvious that in case of changes in the nocturnal pattern of $\mathrm{BP}$, the main problem should be in the regulatory mechanisms, but this hypothesis still needs the proofs.

During puberty, there is an acceleration of changes in BP. Pubertal growth leads to the evaluation of BP that is associated with the increase in gonadal hormones and growth hormone. The interaction of these hormones with several pressor systems may play role in the future risk of hypertension [16]. All this data provide a basis for further study into the relationship between the types of circadian BP profile, parameters of ABPM, hormonal status and cardiovascular health in adolescents.

\section{Conclusion}

Healthy adolescent boys with normal values of both office and diurnal SBP and DBP have different circadian blood pressure patterns. The occurrence of increased BP load was the highest in the group with the nocturnal over-dipping pattern for SBP. The causal mechanism of this is proposed to be complex and an important subject of future research.

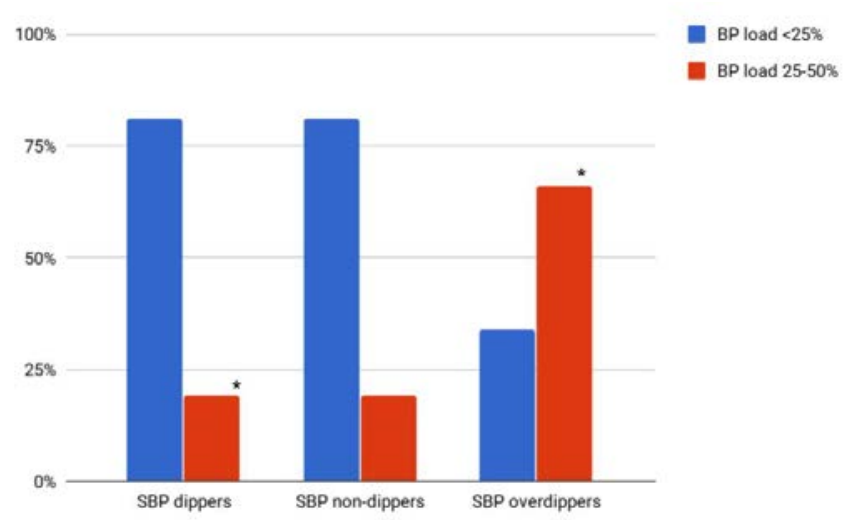

Figure 4. Prevalence of increased BP load in adolescent boys with different circadian SBP pattern.

* The difference was significant, $p<0.05$.

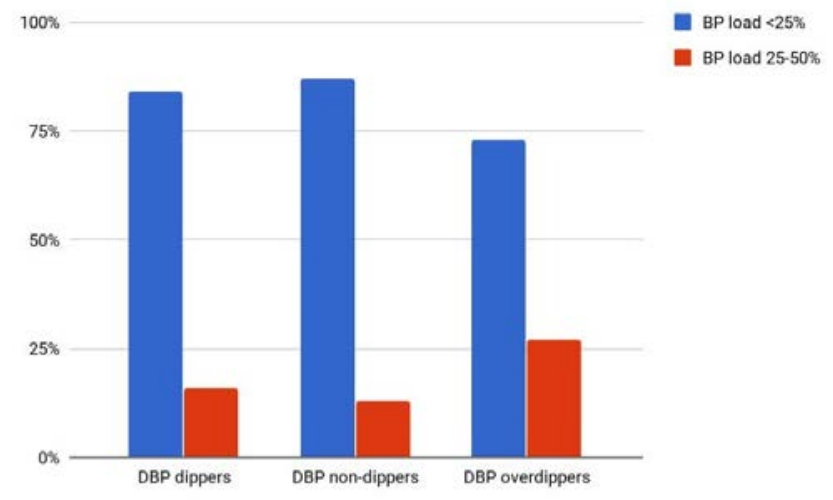

Figure 5. Prevalence of increased BP load in adolescent boys with different circadian DBP pattern.

\section{Conflict of interest}

The authors declare that they have no conflict of interest.

\section{Funding}

This study was funded by the Fund for the promotion of small forms of enterprises in the scientific and technical sphere the program "UMNIK», project №12251GU/2017.

\section{Ethical approval}

All procedures performed in studies involving human participants were in accordance with the ethical standards of the institutional and/or national research committee and with the 1964 Helsinki declaration and its later amendments or comparable ethical standards.

\section{References}

1. Mancia G, Fagard R, Narkiewicz K, Redón J, Zanchetti A, Böhm M, et al. 2013 ESH/ESC Guidelines for the management of arterial hypertension: the Task Force for the management of arterial hypertension of the European Society of Hypertension (ESH) and of the European Society of Cardiology (ESC). J Hypertens 2013; 31(7): 1281-1357. https://doi.org/10.1097/01.hjh.0000431740.32696.cc.

2. White WB, Gulati V. Managing hypertension with ambulatory blood pressure monitoring. Curr Cardiol Rep 2015; 17(2): 2. https://doi.org/10.1007/s11886-014-0556-6. 
3. McCrindle BW. Assessment and management of hypertension in children and adolescents. Nat Rev Cardiol 2010; 7(3): 155-163. https://doi.org/10.1038/nrcardio.2009.231.

4. Gupta D, Chaturvedi S, Chandy S, Agarwal I. Role of 24-h ambulatory blood pressure monitoring in children with chronic kidney disease. Indian J Nephrol 2015; 25(6): 355-361. https://doi.org/10.4103/09714065.148305.

5. Flynn JT, Daniels SR, Hayman LL, Maahs DM, McCrindle BW, Mitsnefes $\mathrm{M}$, et al. Update: Ambulatory Blood Pressure Monitoring in Children and Adolescents. Hypertension 2014; 63(5): 1116-1135. https://doi.org/10.1161/HYP.0000000000000007.

6. Triantafyllidi H, Xenogiannis I, Ikonomidis I, Pavlidis G, Schoinas A, Palaiodimos $L$, et al. The independent relationship of blood pressure load with target organ damage in untreated hypertensive patients: the role of dipping status. Blood Press Monit 2016; 21(6): 366-368. https://doi.org/10.1097/MBP.0000000000000213.

7. Smolensky MH, Hermida RC, Portaluppi F. Circadian mechanisms of 24hour blood pressure regulation and patterning. Sleep Med Rev 2017; 33: 4-16. https://doi.org/10.1016/j.smrv.2016.02.003.

8. Islam MS. Ambulatory blood pressure monitoring in the diagnosis and treatment of hypertension. Adv Exp Med Biol 2017; 956: 109-116. https://doi.org/10.1007/5584 2016 177.

9. Fan HQ, Li Y, Thijs L, Hansen TW, Boggia J, Kikuya M, et al. Prognostic value of isolated nocturnal hypertension on ambulatory measurement in 8711 individuals from 10 populations. J Hypertens 2010; 28(10): 2036-2045. https://doi.org/10.1097/HJH.0b013e32833b49fe.

10. An HR, Park S, Yoo TH, Kang SW, Ryu JH, Lee YK, et al. Non-dipper status and left ventricular hypertrophy as predictors of incident chronic kidney disease. J Korean Med Sci 2011; 26(9): 1185-1190. https://doi.org/10.3346/jkms.2011.26.9.1185.

11. Hermida RC, Ayala DE, Mojón A, Fernández JR. Blunted sleep-time relative blood pressure decline increases cardiovascular risk independent of blood pressure level-the "normotensive non-dipper" paradox. Chronobiol Int 2013; 30(1-2): 87-98. https://doi.org/10.3109/07420528.2012.701127.

12. Ledyaev MY, Stepanova OV, Ledyaeva AM. Validation of the BPlab ${ }^{\circledR} 24$ hour blood pressure monitoring system in a pediatric population according to the 1993 British hypertension society protocol. Med Devices (Auckl) 2015; 8: 115-118. https://doi.org/10.2147/MDER.S78515.

13. Macumber IR, Weiss NS, Halbach SM, Hanevold CD, Flynn JT. The association of pediatric obesity with nocturnal non-dipping on 24-hour ambulatory blood pressure monitoring. Am J Hypertens 2016; 29(5): 647-652. https://doi.org/10.1093/aih/hpv147.

14. Karavanaki K, Kazianis G, Konstantopoulos I, Tsouvalas E, Karayianni C. Early signs of left ventricular dysfunction in adolescents with type 1 diabetes mellitus: the importance of impaired circadian modulation of blood pressure and heart rate. J Endocrinol Invest 2008; 31(4): 289296. https://doi.org/10.1007/BF03346360.

15. Lee $\mathrm{SH}$, Kim JH, Kang MJ, Lee YA, Won Yang S, Shin CH. Implications of nocturnal hypertension in children and adolescents with type 1 diabetes. Diabetes Care 2011; 34(10): 2180-2185. https://doi.org/10.2337/dc11-0830.

16. Shankar RR, Eckert GJ, Saha C, Tu W, Pratt JH. The change in blood pressure during pubertal growth. J Clin Endocrinol Metab 2005; 90(1): 163-167. https://doi.org/10.1210/jc.2004-0926.

Authors:

Anastasia Sheveleva - MD, PhD student, Department of Normal Physiology, Volgograd State Medical Universuty, Volgograd, Russia. https://orcid.org/0000-0001-8276-4757.

Sergey Klauchek - MD, DSc, Professor, Chief of Department of Normal Physiology, Volgograd State Medical University, Volgograd, Russia. https://orcid.org/0000-0003-2206-1894.

Mikhail Ledyaev - MD, DSc, Professor, Chief of Department of Pediatric Disease, Volgograd State Medical University, Volgograd, Russia. https://orcid.org/0000-0003-1414-8789. 